\title{
AUFSÄTZE \\ Experiment Altersöffnung: Politische Ziele und nicht-intendierte Folgen - empirische Befunde aus der Pionierphase des Bundesfreiwilligendienstes
}

\author{
Annelie Beller, M.A. \\ Centrum für soziale Investitionen und Innovationen | Universität Heidelberg | Berlin \\ Research Associate|annelie.beller@csi.uni-heidelberg.de \\ Rabea Haß, M.A. \\ Hertie School of Governance | Berlin | Research Associate \\ hass@hertie-school.org
}

Der Bundesfreiwilligendienst (BFD) ist europaweit der erste klassische Freiwilligendienst - gemessen an Umfang und Verbindlichkeit - der allen Generationen offen steht. Die Altersöffnung wurde als eines von drei notwendigen Elementen angesehen, um eine ausreichende Zahl von Interessenten zu erreichen und den Wegfall des Zivildienstes teilweise zu kompensieren. 18 Monate nach der Reform steigt die Zahl der Interessenten weiter, insbesondere bei der Zielgruppe der über 27-jährigen. Der Beitrag analysiert diese quantitative Entwicklung und beleuchtet auf Grundlage von Fokusgruppeninterviews die Hintergründe, Motivlagen und Erfahrungen der älteren Bundesfreiwilligen. Die Analyse zeigt, dass sich drei Typen unterscheiden lassen: Freiwillige, die den BFD als Qualifizierung sehen; Personen, die den BFD als Alternative zu Erwerbsarbeit oder zu arbeitsmarktpolitischen Maßnahmen betrachten; sowie Freiwillige, die den BFD als Sinnstiftung sehen. Abschließend nimmt der Beitrag eine erste Einordnung vor, inwieweit politisch intendierte Ziele der Altersöffnung erreicht wurden und welche nicht-intendierten Nebenwirkungen aufgetreten sind.

Schlagwörter: Bundesfreiwilligendienst; Altersöffnung; Engagementpolitik; qualitative Sozialforschung; Fokusgruppeninterviews 


\section{Einleitung}

Im Juli 2011 wurde die Wehrpflicht ausgesetzt und damit das Ende des Zivildienstes eingeläutet. Um den Wegfall zu kompensieren, empfahl die Strukturkommission der Bundeswehr um den Chef der Bundesagentur für Arbeit, Frank-Jürgen Weise ,[e]inen freiwilligen, bis zu 23-monatigen Dienst einzuführen, der allen erwachsenen Bürgerinnen und Bürgern offen steht und ihnen die freie Wahl des Engagements bietet“" (Weise u. a. 2010: 28). Die Kommission erörtert weiter „Deutschland kann die Herausforderungen, vor denen wir in den Bereichen Umweltschutz, Katastrophenschutz, Entwicklungshilfe, Pflege, Betreuung und Wohlfahrt stehen, unter den Bedingungen des demographischen Wandels ohne Bürgersinn und zeitgemäßes soziales und bürgerschaftliches Engagement nicht bewältigen“ (Weise u. a. 2010: 28). Bundesministerin Kristina Schröder folgte dieser Empfehlung ${ }^{1}$ und führte den Bundesfreiwilligendienst (BFD) ${ }^{2}$ ein. Das neue Format mit 35.000 Plätzen sollte die zuletzt fast 90.000 Zivildiensteinberufungen pro Jahr, welche im Durchschnitt einer monatlichen Kapazität von 65.794 Zivildienstverhältnissen entsprachen, teilweise ersetzen (Bericht des Bundesbeauftragten für den Zivildienst 2010). Zugleich soll der BFD die Freiwilligenkultur in Deutschland stärken. Strukturell erwächst der Dienst einerseits aus dem staatlich und zentral organisierten Zivildienst. Andererseits knüpft der BFD an die Tradition der Jugendfreiwilligendienste (JFD) an, die sich durch ein diversifiziertes Träger- und Einsatzstellensystem und ein spezifi-

\footnotetext{
${ }^{1}$ Sowohl die Oppositionsparteien SPD und Bündnis 90/Die Grünen als auch die Mehrheit der Wohlfahrtsverbände plädierten eingangs dafür, die Jugendfreiwilligendienste auszubauen statt ein neues Format zu schaffen. Die Verfechter dieses Ansatzes befürchteten, durch den BFD Doppelstrukturen zu schaffen, die JFD zu schwächen und vor allem die zivilgesellschaftlichen Akteure sahen im BFD eine Verstaatlichung, „ein bürokratisches Monstrum, das allen Bemühungen der letzten 20 Jahre, Zivilgesellschaft und bürgerschaftliches Engagement als selbständige Akteure zu stärken, Hohn spricht“" (Strachwitz 2011: 5).

${ }^{2}$ Der Dienst dauert zwischen sechs und 18 Monaten, in Ausnahmefällen bis 24 Monate und wird in der Regel Vollzeit abgeleistet, für Freiwillige ab 27 Jahren $(27+)$ gibt es im BFD Teilzeitstellen mit einer Mindestarbeitszeit von über 20 Wochenstunden. Die Freiwilligen sind sozialversichert und erhalten teilweise eine unentgeltliche Unterbringung, Verpflegung und Arbeitskleidung sowie ein Taschengeld von derzeit maximal 348 Euro monatlich.
} 
sches Bildungskonzept auszeichnen. „Der BFD trifft in Deutschland auf eine ausdifferenzierte und plurale sowie von Eigensinn und Selbstbestimmung geprägte Welt des freiwilligen Engagements, die mit der staatlichen Logik des BFD fremdelt“ (Backhaus-Maul/Nährlich/Speth 2011: 47).

Neben der Herausforderung, diese beiden Kulturen zu verbinden, birgt der Bundesfreiwilligendienst ein weiteres Novum: Er ist europaweit der erste ,klassische' Freiwilligendienst, der allen Generationen offen steht. In Abgrenzung zu anderen Engagementformen ist er durch eine hohe vertraglich geregelte Verbindlichkeit in Bezug auf Umfang und Dauer gekennzeichnet (Jakob 2011: 186). Damit unterscheidet er sich von Pilotprojekten wie dem Freiwilligendienst aller Generationen $(\mathrm{FdaG})$ und dem Generationsübergreifenden Freiwilligendienst (GüF). Auch Frankreich und Italien haben kürzlich staatlich gesteuerte Freiwilligendienste eingeführt, den „Service Civique“ bzw. „Servizio Civile“ (ISS 2011), die Formate stehen jedoch nur jüngeren Freiwilligen offen.

Der vorliegende Beitrag beleuchtet die Altersöffnung des Dienstes und diskutiert diese sowohl aus Sicht der Freiwilligen selbst als auch hinsichtlich der politisch intendierten Zielsetzungen. Grundlage dafür bilden neben Statistiken des Bundesamtes für Familie und zivilgesellschaftliche Aufgaben (BAFzA), Fokusgruppeninterviews mit jüngeren und älteren Freiwilligen sowie Experteninterviews mit Vertretern aus Politik und Drittem Sektor. ${ }^{3}$

\section{Zunehmende Resonanz, auch bei Freiwilligen über 27 Jahren}

Mit der Altersöffnung betrat Deutschland, auch im europäischen Vergleich gesehen, Neuland im Feld der Freiwilligendienste. Die Entscheidung wurde politisch damit begründet, möglichst vielen Personen positive Erfahrungen

${ }^{3}$ Die Datenerhebung fand im Rahmen eines Kooperationsprojektes der Hertie School of Governance und des Centrums für soziale Investitionen und Innovationen (CSI) der Universität Heidelberg statt und wurde von der Gemeinnützigen Hertie-Stiftung finanziert. Erste Ergebnisse daraus wurden im Juli 2012 vorgelegt: Anheier, Helmut K., Annelie Beller, Rabea Haß, Georg Mildenberger und Volker Then (2012): Ein Jahr Bundesfreiwilligendienst. Erste Erkenntnisse einer begleitenden Untersuchung. CSI \& Hertie School of Governance, Berlin. 
durch soziales Engagement zu ermöglichen und einer Altersdiskriminierung entgegenzusteuern. Außerdem wurde die Altersöffnung als eine der „unabdingbare[n] Voraussetzungen für das Erreichen einer zum Erhalt funktionierender Strukturen erforderlichen Zahl von Teilnehmenden“ gesehen (Bundesbeauftragter für den Zivildienst 2010: 20). Dennoch war zu Beginn dieses Experimentes unklar, ob sich ältere Menschen von diesem Dienst überhaupt angesprochen fühlen würden.

Im Januar 2012, also etwa sechs Monate nach Start des BFD, leisteten insgesamt ca. 30.000 Freiwillige ihren Dienst. Davon waren 23 Prozent 27 Jahre und älter. ${ }^{4}$ Seitdem stieg der Anteil der älteren Freiwilligen kontinuierlich, erreichte im April 2012 etwa 30 Prozent und hat sich inzwischen bei ca. 38 Prozent eingependelt. ${ }^{5}$ Die genaue Alterszusammensetzung stellt sich im Januar 2013 folgendermaßen dar (siehe Abb.1).

Auffallend ist, dass es in den ostdeutschen Bundesländern insgesamt mehr Freiwillige $^{6}$ und insbesondere mehr ältere Freiwillige als im Bundesdurchschnitt gibt. Zudem ist der Frauenanteil bei den Freiwilligen ab 27 Jahren deutlich höher (54 Prozent), als bei den Jüngeren (48 Prozent).

${ }^{4}$ Eigene Berechnungen auf Grundlage der Zahlen vom Bundesamt für Familie und zivilgesellschaftliche Aufgaben vom 10. Januar 2012.

5 Laut den Aussagen in den Experteninterviews ist die Nachfrage von älteren Bundesfreiwilligen nach wie vor sehr hoch. Der Anteil könnte also deutlich höher liegen, würde nicht aktiv gegengesteuert. So vergab die Zentralstelle des BAFzA, welche derzeit etwa 14.000 BFD-Plätze verwaltet, im Frühherbst nur noch Plätze an Freiwillige bis 25 Jahre (Kuhlmann 2012: 697).

${ }^{6}$ So leisten im Dezember 2012 in Sachsen 5.034 Personen, in Thüringen 2486 Personen, aber in den bevölkerungsstarken Bundesländern Bayern und BadenWürttemberg nur 3.378 bzw. 4.786 Freiwillige einen BFD. Quelle: BAFzA, Stand 2. Januar 2013. 


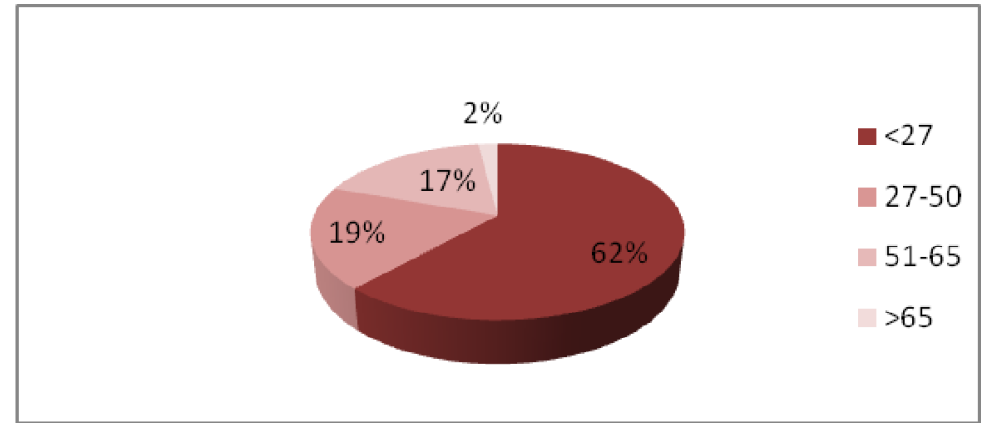

Abb. 1: Prozentuale Altersverteilung, Stand 2. Januar 2013;

Quelle: BAFzA, eigene Berechnungen.

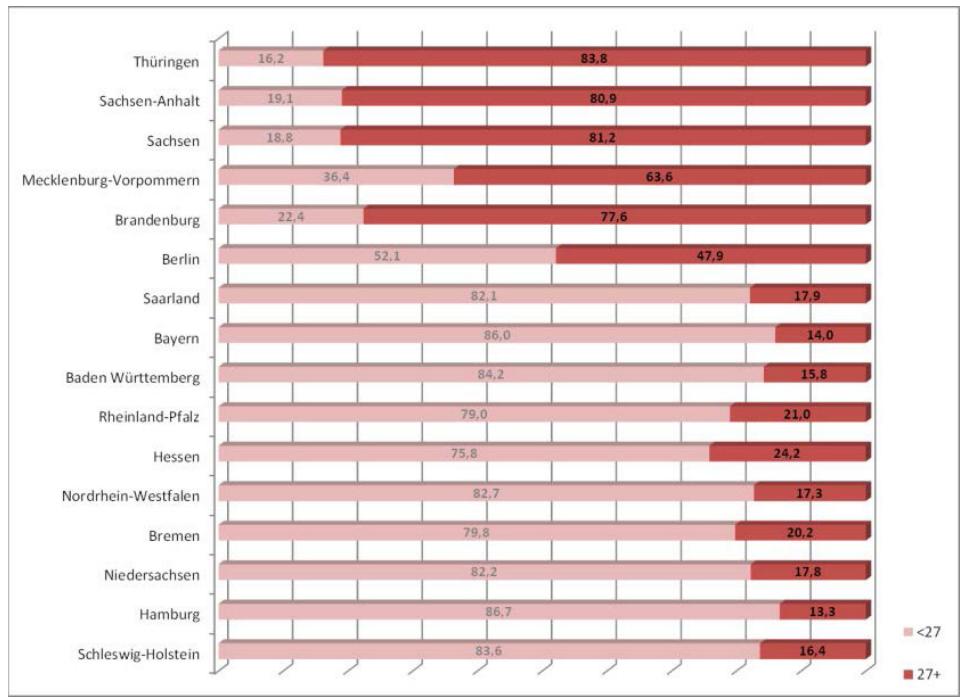

Abb. 2: Altersverteilung nach Bundesländern, Stand 2. Januar 2013;

Quelle: BAFzA, eigene Berechnung.

Wie lassen sich diese regionalen Verteilungsunterschiede erklären? Fest steht auf jeden Fall, dass die unterschiedliche demographische Bevölke- 
rungsstruktur in den neuen und alten Bundesländern die ungleiche Zusammensetzung der TeilnehmerInnen ${ }^{7}$ im BFD nicht erklären kann. Vielmehr spielt die Kombination folgender Faktoren eine Rolle, wie die Auswertung unserer Experteninterviews nahe legt: Der BFD wurde von den regionalen Beratern des BAFzA ${ }^{8}$ unterschiedlich stark beworben, gerade in Sachsen und Thüringen machten sie den Dienst wohl von Anfang an flächendeckend, auch gezielt für ältere Freiwillige, bekannt. Hinzu kamen teilweise sogar Informationsveranstaltungen der Arbeitsagenturen. Zudem haben manche Träger intern unterschiedliche Konzepte für die verschiedenen Dienste entwickelt: Einige beraten die Jüngeren gezielt in Richtung der Ableistung eines FÖJ/FSJ, die Älteren hingegen in Richtung BFD. Und schließlich spielen Arbeitsmarktstrukturen eine Rolle: Für viele Arbeitssuchende ist der Dienst eine attraktive Alternative zum Arbeitsmarkt oder arbeitsmarktpolitischen Maßnahmen.

\section{Im Fokus: Hintergründe, Motive und Erfahrungen der Zielgruppe 27+}

In Fokusgruppeninterviews an bundesweit neun verschiedenen Standorten bei unterschiedlichen Trägern haben wir 164 Freiwillige befragt. Die Fokusgruppen fanden im Rahmen der Seminare statt. Die Teilnahme daran war freiwillig und erfolgte anonym. Freiwillige unter und über 27 Jahre wurden getrennt befragt. Die Fokusgruppen wurden als leitfadenorientierte Gruppeninterviews organisiert, in deren Mittelpunkt die Themen Motivation und Entscheidung für den BFD, bisherige Erfahrungen in den Einsatzstellen und den Seminaren sowie die jeweiligen Pläne für die Zeit nach dem Dienst standen. Die Interviews wurden aufgezeichnet, transkribiert und im An-

\footnotetext{
${ }^{7}$ Im Folgenden wird zugunsten einer besseren Lesbarkeit ausschließlich die männliche Form verwendet. Dies schließt aber stets beide Geschlechter mit ein.

${ }^{8}$ Die ehemaligen Regionalbetreuer des Zivildienstes sind nun als Vertreter des BAFzA die regionalen Ansprechpersonen für alle Freiwilligen sowie Einsatzstellen und Träger, die keiner Zentralstelle angehören. Zu ihren Aufgaben gehören die Beratung und Betreuung der Freiwilligen und Einsatzstellen während des Dienstes sowie hinsichtlich der pädagogischen Begleitung im BFD.
} 
schluss inhaltsanalytisch ausgewertet. Zudem füllten die Freiwilligen einen Kurzfragebogen aus, in dem soziodemografische Daten (Alter, Geschlecht, Status vor dem BFD) abgefragt wurden.

Von den insgesamt 164 Befragten waren 87 Freiwillige 27 Jahre und älter, das entspricht 53 Prozent. Insgesamt gliedern sich die Fokusgruppenteilnehmer wie folgt in die verschiedenen Altersgruppen auf:

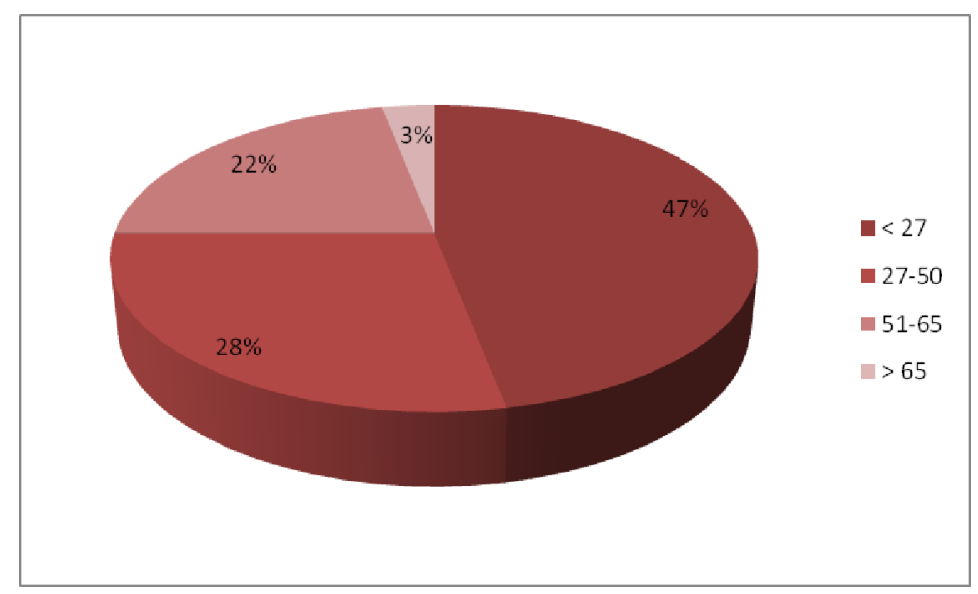

Abb. 3: Altersverteilung der Fokusgruppenteilnehmer in Prozent;

Quelle: Eigene Erhebung und Berechnung.

Bei den Fokusgruppenteilnehmern 27+ entspricht die Altersstruktur in etwa dem Bundesdurchschnitt (vergleiche Abbildung 1): 28 Prozent entfallen auf die Altersgruppe 27 bis 50; 22 Prozent sind zwischen 51 und 65 Jahre alt. Personen über 65 Jahre finden sich mit drei Prozent nur vereinzelt wieder. ${ }^{9}$ Insgesamt nahmen 67 weibliche und 97 männliche Freiwillige an den Fokusgruppen teil.

Ähnlich wie im bundesweiten Gesamtbild ist auch in den Fokusgruppen der Anteil der weiblichen Freiwilligen bei den älteren Teilnehmern höher als bei den jüngeren. Bezüglich ihrer Tätigkeiten vor dem BFD unterscheiden sich die Altersgruppen eklatant. Die Teilnehmer bis 27 Jahre kamen bis auf

\footnotetext{
${ }^{9}$ Die Personen über 65 Jahre waren erwartungsgemäß alle in Rente.
} 
wenige Ausnahmen direkt von der Schule. Einige wenige traten den BFD nach einer Ausbildung oder einem Studium an. Nur ein Freiwilliger unter 27 Jahren war vor dem Dienst arbeitssuchend. ${ }^{10}$

In der Altersgruppe 27-65 hingegen waren 60 von 82 Teilnehmern vor dem BFD arbeitssuchend. Sämtlich bezogen sie Arbeitslosengeld II (ALG II). Zehn Personen in dieser Altersgruppe gingen vorher einer Beschäftigung nach, wobei je ein Teilnehmer Ausbildung, Studium bzw. Rente als vorherige Beschäftigung angab. Die restlichen neun Teilnehmer dieser Altersgruppe kreuzten „Sonstiges“"11 als Status vor dem BFD an.

$\mathrm{Zu}$ Beginn unserer Untersuchung erwarteten wir, dass sich aufgrund der Rahmenbedingungen des Dienstes nur bestimmte Personenkreise für den Dienst entscheiden würden:

- Personen, die bei ihren Eltern wohnen, also auf „Taschengeldniveau“ leben können;

- Personen, die durch privates oder familiäres Vermögen abgesichert sind;

- Personen, die vom Arbeitgeber für den Dienst frei gestellt werden und zumindest teilweise ihr Gehalt bzw. ihren Lohn weiter beziehen;

- Personen, die durch Lebensgefährten mitfinanziert werden, zum Beispiel ein Elternteil nach der Familienphase;

- Personen, deren Lebensunterhalt durch Rentenbezüge oder Pensionsansprüche gesichert ist;

- Personen, für die der BFD eine (finanzielle) Statusverbesserung bedeutet, beispielsweise durch das teilweise anrechnungsfreie Taschengeld zu ALG II-Bezügen oder als Aufstockung zu geringen Rentenbezügen;

\footnotetext{
${ }^{10}$ Von den 77 Teilnehmern bis 27 Jahren kamen drei Personen aus einer Ausbildung, zwei kamen jeweils aus Job oder Studium, eine Person war arbeitssuchend.

${ }^{11}$ Manche ergänzten diese Angabe mit dem Hinweis auf Bürgerarbeit, AGH-MAE (Arbeitsgelegenheiten mit Mehraufwandsentschädigung) oder 1-Euro-Job, so dass davon ausgegangen werden kann, dass es sich hier teilweise um ALG II-Empfänger handelt.
} 
- Personen, die sich davon einen Mehrwert für ihre berufliche Zukunft erhoffen, so dass sie die finanziellen Einschränkungen während des Dienstes als (Bildungs-)Investition sehen (Anheier u. a. 2012: 12).

Die Angaben der Fokusgruppenteilnehmer bestätigen diese Annahmen nun. Aus den soziodemographischen Daten können wir ableiten, dass die überwiegende Zahl der älteren Freiwilligen im erwerbsfähigen Alter ist. Zudem zeigt sich, dass 73 Prozent der Freiwilligen zwischen 27 und 65 vor Antritt des BFD arbeitssuchend (ALG II-Empfänger) waren. Das lässt den Schluss $\mathrm{zu}$, dass die Freiwilligen keine bewusste Auszeit aus dem Erwerbsleben nehmen, sondern vielmehr aus Qualifizierungsgründen oder aber um eine Statusverbesserung zu erreichen, am BFD partizipieren. Dies deckt sich mit den Ergebnissen einer Befragung des Wohlfahrtswerkes für BadenWürttemberg, wonach 68 Prozent der älteren BFDler vor Dienstantritt arbeitssuchend bzw. als 1-Euro-Jobber tätig waren (Gölz 2012: 214). Darin sehen wir auch die Tatsache begründet, dass die Freiwilligen, befragt nach ihren Motiven, den BFD vor allem als Alternative zur Erwerbsarbeit oder zu anderen Beschäftigungsformen (1-Euro-Job, Bürgerarbeit, etc.) sehen und das Taschengeld, welches bis zu 175 Euro anrechnungsfrei auf ALG II ist (Stand: Januar 2013), oft als finanzieller Anreiz genannt wird. Die Freiwilligen nennen zudem weitere Vorteile: Die Tätigkeit kann selbst ausgesucht werden, sie wird als sinnvoll erachtet - v.a. im Gegensatz zu Arbeitsgelegenheiten mit Mehraufwandsentschädigung (AGH-MAE) oder verpflichtende Weiterbildungen durch die Arbeitsagenturen - und macht Spaß. In der Gesamtanalyse der Fokusgruppen wurden neben der Alternative zur Erwerbsarbeit und dem finanziellen Anreiz die folgenden fünf Motive am häufigsten genannt: reizvolle Tätigkeit, Möglichkeit zur Intensivierung des Ehrenamtes, berufliche Neuorientierung, der Gesellschaft etwas zurückgeben sowie gesellschaftliche Integration und Teilhabe:

„Ich konnte nicht mehr hören, dass doch diese Hartz IV-Empfänger gefälligst irgendwas für die Gesellschaft tun sollten. [...] Dann bin ich zum Job-Center gegangen und habe gesagt, dass ich jetzt im Park Papier sammle. Und dann haben die gesagt: So einfach geht das nicht und dadurch ergab sich der BFD. So, und jetzt mach ich was für die Gesellschaft und jetzt können sie mich gefälligst in Ruhe lassen." (Fokusgruppenteilnehmer 27+) 
„Den BFD haben wir gemacht, weil wir keine andere Möglichkeit hatten neben Hartz IV noch einen Erwerb zu haben. Also, vom Arbeitsamt kam nichts rüber, da [gab es] nur noch den Bundesfreiwilligendienst. Und das hat man durch dritte Personen erfahren." (Fokusgruppenteilnehmer $27+$ )

Es zeigt sich also, dass nebst intrinsischer Motivation auch gesamtgesellschaftliche und arbeitsmarktpolitische Entwicklungen eine Rolle spielen. Viele ältere Freiwillige sehen im BFD eine Alternative zur Erwerbsarbeit.

Es zeichnet sich somit ab, dass sich die Motive der Älteren deutlich von den Jüngeren unterscheiden. Die jüngeren Freiwilligen nannten in der Evaluation von FSJ und FÖJ vor allem die Beweggründe Berufsorientierung, Persönlichkeitsentwicklung und das Sammeln von Praxiserfahrung (Engels/Leucht/Machalowski 2005: 148). In unseren Fokusgruppen zeichnet sich bei den Freiwilligen bis 27 Jahren ein ähnliches Bild ab; hier unterscheidet sich der BFD also nicht von den etablierten JFD.

\section{Drei Typen von Freiwilligen in der Altersgruppe 27+}

In den Gruppendiskussionen stellen die Teilnehmer ihre Erfahrungen unabhängig von ihren Motiven und Erwartungen zum größten Teil als sehr positiv dar, vor allem das Gefühl „gebraucht zu werden“. Viele Freiwillige beschreiben insbesondere von den Empfängern ihrer unterschiedlichen Dienstleistungen viel Anerkennung und Dankbarkeit zu erfahren:

„Und es ist schön, dass man jeden Tag an die Arbeit gehen kann; dass man Leuten begegnet; man sich unterhalten kann und austauschen kann, das ist das Schönste dabei und nicht in den vier Wänden sitzt und an seine Wände [an]starrt.“ (Fokusgruppenteilnehmer 27+)

Neben diesem grundsätzlich positiven Fazit unterscheiden sich die weiteren Erfahrungen und Erwartungen der älteren Freiwilligen. Es lassen sich drei Typen identifizieren, welche stark mit den Motiven und biographischen Lebensphasen verbunden sind: 1) Freiwillige, die den BFD aus Qualifizierungsgründen absolvieren, 2) Freiwillige, die den Dienst primär als eine Alternative zum Arbeitsmarkt sehen und 3) Freiwillige, die den BFD als sinnstiftende Aufgabe ansehen und meist bereits im Ruhestand sind. Im Folgenden werden die drei Typen näher beschrieben. 


\subsection{Typ 1: BFD als Qualifizierung}

Freiwillige des ersten Typus betonen stärker als die anderen beiden Typen, die Chancen, die sie im Dienst sehen. Für sie liegt der Fokus auf einem Ausbildungsabschnitt mit einer relativen Sicherheit, dass danach ein weiterer Ausbildungs- oder Weiterbildungsabschnitt folgt. $\mathrm{Zu}$ diesem Personenkreis gehören neben Freiwilligen im Anerkennungsjahr auch ausländische Freiwillige, die einen Einstieg in den deutschen Arbeitsmarkt suchen oder Personen, die sich beruflich neu orientieren wollen.

„Jetzt gehe ich morgens um acht in die Schule, komme abends um sieben heim, ich bin komplett fertig, aber meine Frau sagt: ,Du hast so ein Leuchten in den Augen'. Es ist so eine tiefe innere Zufriedenheit, die ich jetzt spüre und ich bin einfach nur zufrieden. Das persönliche Ziel ist jetzt einfach, ich möchte nächstes Jahr dann Sozialpädagogik studieren.“ (Fokusgruppenteilnehmer 27+)

\subsection{Typ 2: BFD als Alternative zur Erwerbsarbeit oder arbeitsmarkt- politischen Maßnahmen}

Die Reflexionen der Freiwilligen, die den Dienst vor allem als Alternative zur Erwerbsarbeit ansehen, und in den Fokusgruppen zahlenmäßig überwogen (siehe dazu auch Gölz 2012: 214), zeichnen ein deutlich kritischeres Bild. Nicht über die Tätigkeit selbst, aber die strukturellen Rahmenbedingungen. Auffallend ist, dass sich diese Freiwilligen stark mit den hauptamtlichen Mitarbeitern ihrer Einsatzstellen vergleichen, nicht aber mit anderen Ehrenamtlichen, die sich außerhalb eines Freiwilligendienstformates engagieren. Bedingt durch diesen Vergleich geben einige Freiwillige in den Fokusgruppen subjektive Erlebnisse über Stigmatisierungen und Diskriminierungen wieder. Sie legen dar, sich als Mitarbeiter zweiter Klasse zu fühlen:

„Wenn jemand [in meiner Einsatzstelle] am Wochenende arbeitet, dann ist man Mitarbeiter, aber wenn [es] dann eine Bezugssache ist, dann ist man nur ein Freiwilliger.“ (Fokusgruppenteilnehmer 27+)

Der Großteil der Teilnehmer würde den Dienst anderen Personen weiterempfehlen, am liebsten jedoch selbst noch länger weitermachen. Bei einem Träger, bei dem nach einem Jahr die Möglichkeit bestand, auf die maximalen 18 Monate Dienstzeit zu verlängern, haben 80 Prozent diese Chance 
wahrgenommen. Auch dieser Befund unterstreicht die These, dass der BFD für viele die einzige Alternative zur Erwerbsarbeit darstellt und nicht primär als Passage, Orientierungs- oder Bildungsjahr genutzt wird.

Dem Ende des Dienstes sieht dieser Typus von Bundesfreiwilligen besorgt entgegen, da viele noch keine Anschlussperspektive haben. Die oftmals implizit erhoffte Anschlussbeschäftigung, auch wenn kein einziger Freiwilliger davon berichtete, dass eine solche zu Beginn des Dienstes von den Organisationen in Aussicht gestellt worden wäre, erfüllt sich somit für viele nicht. Hingegen bieten Einsatzstellen vereinzelt an, weiterhin entweder ehrenamtlich (ohne finanziellen Anreiz) oder, in Ausnahmefällen, auf Grundlage einer Aufwandsentschädigung tätig zu sein. Insgesamt erfahren kurz vor Ende des Dienstes nur wenige eine aktive Unterstützung durch die Einsatzstellen bei der Suche nach einer Folgebeschäftigung, sei es im ehrenamtlichen Bereich oder in hauptamtlichen Strukturen. Hier wird erkennbar, dass sich einige Einsatzstellen noch nicht auf die neue Klientel eingestellt haben. Denn in den JFD und im Zivildienst stellte sich die Frage, wie es danach weitergehen würde, in der Regel nicht: ein Großteil der jüngeren Freiwilligen oder ehemaligen „Zivis“ begann im Anschluss eine Ausbildung oder ein Studium, oft sogar in einer anderen Stadt. Hier birgt die Altersöffnung grundsätzlich eine Chance für die Einsatzstellen, die älteren Freiwilligen durch attraktive Angebote langfristig zu binden, die, den Angaben in unseren Experteninterviews zu Folge, bisher noch kaum genutzt wird.

In den Fokusgruppen zeichnet sich also die Tendenz ab, dass dieser Typus den Dienst analog zu arbeitsmarktpolitischen Maßnahmen beschreibt:

„Ich arbeite da schon acht Jahre. Erst ABM, MAE, dann ehrenamtlich und jetzt dieses BFD.“ (Fokusgruppenteilnehmer 27+)

\subsection{Typ 3: BFD als sinnstiftendes Format}

Rentner, die sich aus unterschiedlichen Motiven für diese Engagementform entscheiden, bilden den dritten Freiwilligentypus in den Fokusgruppen. Diese Gruppe berichtet größtenteils über positive Erfahrungen, vor allem dahingehend ,nicht mehr alleine zuhause zu sitzen “ und ihre Freizeit sinnvoll gestalten zu können. Quantitativ sind jedoch die wenigsten Freiwilligen, 
nämlich drei Prozent aus den Fokusgruppen und zwei Prozent im Bundesdurchschnitt, dieser Gruppe zuzuordnen. ${ }^{12}$

Über alle Typen hinweg werden Schwierigkeiten bei der Integration in die organisationalen Abläufe beschrieben. Dies betrifft insbesondere den unklaren Status der Freiwilligen in den Organisationen und sich daran anschließende offene Fragen, beispielsweise die Teilnahme an Teambesprechungen, aber auch Feiertags-, Wochenend- und Urlaubsregelungen. In den Fokusgruppen mit den Jüngeren werden diese Punkte von den Freiwilligen nicht thematisiert.

\section{Politisch intendierte Ziele und nicht-intendierte Nebenwirkungen}

Die langfristigen Effekte ${ }^{13}$ auf die Biographien der Freiwilligen werden erst in einigen Jahren erfasst werden können. Dennoch kann eine erste Einordnung unternommen werden, inwieweit sich die politischen Intentionen des Bundesfreiwilligendienstes erfüllen, gerade in Hinblick auf die Altersöffnung.

Bei diesem Abgleich zeichnen sich nicht-intendierte Folgen der Reform ab, die in engem Zusammenhang mit der neuen Zielgruppe 27+ stehen. Um auch hier den Ansprüchen der Reform voll gerecht zu werden und die positiven Ansätze im BFD langfristig nicht zu überschatten, bedarf es einer klugen Feinsteuerung.

\footnotetext{
${ }^{12}$ Grundsätzlich könnte dieser Typus auch Personen umfassen, die im berufsfähigen Alter sind, durch ihren Ehepartner oder eigenes Vermögen finanziell abgesichert sind und sich als „Zeitvertreib“ für einen BFD entscheiden. In unseren Fokusgruppen waren solche Teilnehmer jedoch nicht vertreten und auch die Experteninterviews deuten darauf hin, dass sich diese Personen nur sehr vereinzelt für das Format BFD mit seiner hohen Verbindlichkeit entscheiden.

${ }^{13}$ Die Institute INBAS-Sozialforschung, INBAS und ISG führen im Auftrag des BMFSFJ die Gemeinsame Evaluation des Gesetzes über den Bundesfreiwilligendienst (BFDG) und des Gesetzes zur Förderung von Jugendfreiwilligendiensten (JFDG) durch. In einer Längsschnittbefragung werden Freiwillige von Herbst 2012 bis Herbst 2014 zu drei Zeitpunkten befragt. Erste Zwischenergebnisse werden Ende 2013 erwartet. Die Gesamtergebnisse und Handlungsempfehlungen der Evaluation sollen im Herbst 2015 vorliegen.
} 
Folgende drei Ziele stehen im Mittelpunkt der Reform:

1) Negative Folgen der Aussetzung des Zivildienstes minimieren: Im Gesetzesentwurf der Bundesregierung von Februar 2011 heißt es: „Ziel des Gesetzes ist es, diese negativen Effekte zu minimieren, damit auch zukünftig möglichst viele Menschen durch soziales Engagement positive Erfahrungen sammeln können“ (Deutscher Bundestag 2011: 1).

2) Kultur der Freiwilligkeit stärken und Anerkennungskultur verankern: Das Bundesministerium für Familie, Senioren, Frauen und Jugend (BMFSFJ) sieht den „Bundesfreiwilligendienst [als] ein engagementpolitisches Projekt: Er soll eine neue Kultur der Freiwilligkeit in Deutschland schaffen" (BMFSFJ 2012b). Zudem wird die Anerkennungskultur besonders betont: „Menschen, die anderen Menschen ihre Zeit schenken und sich für das Gemeinwohl engagieren, sollen dafür auch die Wertschätzung bekommen, die sie verdienen“" (Schröder 2012: 687).

3) Lebenslanges Lernen: Der Bundesfreiwilligendienst ist eine besondere Form des Engagements. Zeitlich begrenzt und in einen gesicherten Rechtsrahmen eingebettet (Schröder 2012: 686), charakterisiert $\S 1$ des BFDG den Dienst als Lerndienst.

\subsection{Intention 1: Folgen des Endes des Zivildienstes minimieren}

Die quantitative Entwicklung zeigt, dass die 35.000 Plätze, die im BFD zur Verfügung stehen, innerhalb des ersten Jahres besetzt werden konnten. Insbesondere die Altersöffnung dürfte hier eine wichtige Rolle gespielt haben, auch wenn die Altersgruppe der über 65-jährigen eher hinter den Erwartungen zurückbleibt, wohingegen von den „mittleren“ Altersgruppen (27-65) eine höhere Nachfrage ausgeht als anfänglich vermutet. ${ }^{14}$ In Anbetracht des demographischen Wandels und den guten Aussichten auf dem Ausbildungsmarkt in den kommenden Jahren, die zu einer niedrigeren Nachfrage bei jungen Freiwilligen führen könnten (Kuhlmann 2012: 698), wird

\footnotetext{
${ }^{14}$ Das ergab die Auswertung unserer 21 Experteninterviews, die wir zwischen Dezember 2011 und Januar 2013 mit Entscheidungsträgern auf Ebene des BMFSFJ, des BAFzA sowie der Zentralstellen und Träger im BFD geführt haben.
} 
die Altersöffnung auch in Zukunft für ein konstantes Interesse am Dienst sorgen.

Auch wenn die neu geschaffenen Plätze in BFD und JFD die zuletzt 90.000 Zivildienstleistenden nicht gänzlich ersetzen können und sollten, so ist die befürchtete Versorgungslücke ${ }^{15}$ doch ausgeblieben. Der BFD hat das Ziel, die negativen Folgen der Aussetzung des Zivildienstes zu minimieren, somit erreicht.

\title{
5.2. Intention 2: Kultur der Freiwilligkeit stärken und Anerkennungs- kultur verankern
}

Auf den ersten Blick spricht die hohe Zahl der Interessenten am BFD für eine gut etablierte Kultur der Freiwilligkeit in Deutschland. Menschen aller Altersgruppen sind offensichtlich bereit, sich gegen ein Taschengeld für das Allgemeinwohl einzusetzen. Bei näherer Analyse der Zielgruppe 27+ und deren biographischen Hintergründen, drängt sich jedoch die Frage auf, inwiefern das Engagement wirklich freiwillig ist.

\begin{abstract}
„Also freiwillig, tatsächlich freiwillig macht man das nur, wenn man genügend Kleingeld hat, wenn die eigene Existenz gesichert ist. Die Freiheit beginnt oberhalb 1.000 Euro monatlich für eine Person. Darunter liegen Engpässe, Nöte und Abgründe. Die gibt es zwischen Null und Tausend in allerlei Varianten. Das Ausmaß der Nötigung steigt mit dem Sinken des Einkommens.“ (Gewerkschaftsvertreter)
\end{abstract}

In den Fokusgruppen schildern ältere Freiwillige immer wieder, sich als Mitarbeiter zweiter Klasse wahrzunehmen. Sie bekommen zwar von den Menschen, die ihre Dienstleistungen empfangen, Anerkennung und positive Rückmeldungen, und ihre Arbeit wird auch in den Einsatzstellen dankbar angenommen, jedoch nur unter der Prämisse, dass sie freiwillig und ohne reguläres Gehalt erbracht wird. Dies könnte zu einer „Dequalifizierung Sozialer Arbeit“(Jirku 2011: 74) führen, wobei versucht wird, „Lücken in gemeinwohlorientierten Arbeitsmärkten so billig wie möglich zu schließen“ (Jirku 2011: 74). In der Folge könnte dies zu weiterer Unzufriedenheit führen und ein oftmals schon vorhandenes Minderwertigkeitsgefühl verstärken.

\footnotetext{
${ }^{15}$ So befürchteten beispielsweise die Wohlfahrtsverbände in Bayern Engpässe, wie die Süddeutsche Zeitung am 10. Juli 2011 berichtete (Szymanski 2011).
} 
„Ja, Bufdi, das ist einer, der jetzt gerade keinen Job hat, und der geht halt sozial arbeiten. [...] ...weil, da schäme ich mich, muss ich wirklich sagen. Das war für mich nach außen hin ein sozialer Abstieg.“ (Teilnehmer Fokusgruppe 27+)

Hier zeichnet sich also eine nicht-intendierte Entwicklung gerade durch die Altersöffnung ab, die auch die Befragung des Wohlfahrtswerkes für BadenWürttemberg unterstreicht, nach deren Ergebnis sich 44 Prozent der älteren Freiwilligen nicht oder nur in geringem Maße gesellschaftlich anerkannt fühlen (Gölz 2012: 215).

Teilweise mag das dem Umstand geschuldet sein, dass der BFD auf Grund seiner kurzen Existenz noch nicht jedem bekannt ist. Zugleich darf in diesem Zusammenhang jedoch nicht verklärt werden, dass die Nähe zu arbeitsmarktpolitischen Instrumenten dem Format BFD einen nachhaltigen Imageschaden zufügen könnte. Denn zeitgleich mit der Einführung des Dienstes wurden andere Instrumente wie 1-Euro-Jobs gekürzt oder ersatzlos gestrichen und durch die Altersöffnung bietet sich der BFD genau für diese Personen nun als Ersatz an. Der Bundestagsabgeordnete Ulrich Schneider (Bündnis 90/Die Grünen) summiert diese Entwicklung wie folgt:

„Der Grat zwischen Arbeitsmaßnahme und freiem Entschluss des Einzelnen ist schmal. Insbesondere die erst erfolgte Instrumentenreform mit massiven Kürzungen zur Förderung und Weiterbildung Arbeitssuchender könnte auf die Entwicklungen im BFD ebenfalls Auswirkungen haben“" (Schneider 2013).

Zum einen kann dies dem Ansehen des BFD und den Freiwilligendiensten im Allgemeinen abträglich sein, zum anderen führt diese Koinzidenz von Maßnahmenkürzungen und Einführung des BFD dazu, dass teilweise eben genau diese Personen, die von Kürzungen betroffen waren, in den Einsatzstellen unter dem neuen Label BFD übernommen wurden. Diese Umwidmung trägt dazu bei, dass sich nicht - wie politisch intendiert - neue Gesell- 
schaftsschichten engagieren, sondern vielerorts bereits Aktive ihr Engagement umwidmen oder intensivieren. ${ }^{16}$

Gerade deshalb ist es wichtig, dieses besondere Dienstformat mit seinem spezifischen gesetzlichen Rahmen von arbeitsmarktpolitischen Maßnahmen, Erwerbsarbeit und anderen Formen des bürgerschaftlichen Engagements klar abzugrenzen. Ein Alleinstellungsmerkmal könnte dabei die Ausgestaltung als Lerndienst sein.

\subsection{Intention 3: Ein Lerndienst für alle Generationen}

Das BFD-Gesetz gibt vor, dass der Dienst für alle Generationen als Lerndienst ausgestaltet werden soll. Dabei stehen die beiden Säulen „Pädagogische Begleitung in den Einsatzstellen“ und „Begleitende Seminare“ im Fokus. ${ }^{17}$ Während die Bildung für die jüngeren Freiwilligen eng an die Standards aus FSJ und FÖJ angelehnt wurde, sieht das Gesetz für die älteren Freiwilligen eine Seminarteilnahme in ,angemessenem Umfang“ vor (BFDG §4 (3)). Seit Ende 2012 gilt die nicht bindende Richtlinie des BAFzA, dass ein Tag pro Dienstmonat für diese Altersgruppe angemessen sei. Auf Grund der Interpretationsspielräume im Gesetz und der bisher geringen Erfahrung mit älteren Freiwilligen ist der BFD bisher nur für Freiwillige bis 27 Jahren ein Lerndienst; bei der Zielgruppe 27+ erreicht das Bildungsangebot aus unterschiedlichen Gründen bei weitem noch nicht alle.

Dr. Jens Kreuter, Leiter des Arbeitsstabes Freiwilligendienste im BMFSFJ, betont den hohen Stellenwert der informellen Lernprozesse im Alltag der Freiwilligen: „Jeder Freiwilligendienst wird durch die Erfahrung im täglichen Engagement zu einem prägenden Erlebnis und einem wesentlichen Teil informeller Bildung“ (Kreuter 2012: 1). Auch wenn diese Lernkomponente sicher wichtig ist, so reicht sie nicht aus, um den besonderen Merkmalen der Engagementform „Freiwilligendienst“ gerecht zu werden. Bisher fällt es vielen verantwortlichen Trägern und Einsatzstellen schwer, den Bildungsauftrag zu erfüllen. Die Gründe hierfür sind vielfältig. Zum einen ist die

\footnotetext{
${ }^{16}$ Siehe dazu auch die Erfahrungen aus dem Workshop Praxiserfahrungen mit „Ü 27“ der Fachtagung ,Die neue Kultur der Freiwilligkeit - Perspektiven der Freiwilligendienste in Deutschland“ (BMFSFJ 2012a:. 11).

${ }^{17}$ Vgl. BFDG § 1: „Der Bundesfreiwilligendienst fördert lebenslanges Lernen“ und $\S 4$ definiert den Umfang der pädagogischen Begleitung.
} 
Klientel der älteren Freiwilligen deutlich heterogener was ihre Lebenserfahrung, Erwartungen und Bildungshintergründe angeht. Zudem sehen sich die Akteure mit Vorurteilen konfrontiert: Die älteren Freiwilligen sehen teilweise den Sinn der Seminare nicht, verbinden diese Begrifflichkeit mit Schule oder haben Angst, was dort auf sie zukommt; in Einzelfällen können sie kaum lesen und schreiben; andere können das Angebot aus organisatorischen Gründen nicht wahrnehmen, sei es, weil sie ein Kind oder einen pflegebedürftigen Angehörigen zu Hause betreuen müssen und daher nicht ganztags oder sogar mehrere Tage an einem Seminar teilnehmen können; oder weil sie beispielsweise im ländlichen Raum ohne eigenen PKW und schlechter Verkehrsanbindung wohnen. ${ }^{18}$ Die Bildungsangebote gerade für die älteren Freiwilligen verbindlich zu machen birgt jedoch neben den genannten strukturellen Hürden ein zusätzliches Dilemma:

„Und im Ehrenamt oder bürgerschaftlichen Engagement ist eben diese Freiwilligkeit auch wirklich freiwillig. Bei den unter 27-jährigen ist die Bildungsarbeit, obwohl es freiwillig ist, natürlich nicht freiwillig, sondern es sind vorgeschriebene Seminare. Da gibt es einen Zwang, mithin, bis hin zur Restriktion. Wir hatten alle große Hemmungen, also alle nicht, aber ein guter Teil hatte Hemmungen, eben hier diesen Zwang wieder zu machen - also die Vorstellung, einem 70-jährigen zu erklären: ,Du musst jetzt an einem Seminar teilnehmen, ansonsten komme ich vorbei und ziehe Dir die Ohren lang oder ich drohe Dir oder nehme Dir irgendwas weg', war uns zutiefst suspekt.“(Verbandsvertreter)

Ohne diesen Zwang werden aber viele der älteren Freiwilligen nicht erreicht. Selbst in Verbänden, deren Bildungsangebot für die Zielgruppe 27+ bereits flächendeckend besteht und die jeden Freiwilligen persönlich einladen, schätzen Verbandsvertreter in den Experteninterviews die Teilnehmerquote auf etwa ein Drittel bis maximal 50 Prozent. In diesem Bereich entsteht also eine neue Herausforderung durch die Zielgruppe 27+.

${ }^{18}$ Diese Einschätzung beruht sowohl auf den durchgeführten Experteninterviews, Beobachtungen während der Fokusgruppen sowie den Erfahrungen aus dem Workshop „Pädagogische Begleitung von über 27-Jährigen“ im Bundesfreiwilligendienst der Fachtagung ,Die neue Kultur der Freiwilligkeit Perspektiven der Freiwilligendienste in Deutschland“ (BMFSFJ 2012a: 13ff). 
Viele pädagogische Mitarbeiter setzen darauf, dass die Vorbehalte und Ängste gegenüber den Seminaren durch Mund-zu-Mund-Propaganda langsam abgebaut werden können. Denn die Freiwilligen, die einmal an einem Bildungsangebot teilgenommen haben, sind in der Regel sehr zufrieden und kommen bei der nächsten Einladung wieder, so die Einschätzung der befragten Experten.

Die Notwendigkeit, den BFD als Lerndienst zu stärken, unterstreichen auch folgende Gesichtspunkte:

- Die Komponente des Lerndienstes ist ein wichtiges Abgrenzungsmerkmal zu arbeitsmarktpolitischen Maßnahmen, Erwerbsarbeit, beispielsweise einem 400-Euro-Job, und „normalem“ ehrenamtlichen Engagement.

- Nur durch flächendeckende und verbindliche Einführungsseminare kann sichergestellt werden, dass jeder Freiwillige über seine Rechte und Pflichten hinreichend aufgeklärt wird.

- Durch die fehlenden Bildungskonzepte für die Zielgruppe 27+ können schnell Qualitätsstandards untergraben werden, was sich negativ auf alle Freiwilligendienste auswirken könnte (Anheier u. a. 2012: $18)$.

Die gewährten Freiräume im Gesetz über den Bundesfreiwilligendienst (BFDG) hatten die Intention, einen Experimentier- und Gestaltungsspielraum gerade für die neue Zielgruppe $27+$ zu eröffnen, weil anfänglich nicht abzusehen war, in welchem Umfang und aus welcher Motivation sich ältere Freiwillige für den BFD bewerben würden. Nun könnten genau diese Interpretationsspielräume den Einsatzstellen Vorschub leisten, die ihre Freiwilligen möglichst gewinnbringend einsetzen möchten, indem sie den älteren Freiwilligen keine oder nur sehr wenige Seminare anbieten. Auch der monatliche Zuschuss von 200 Euro pro Freiwilligen, den das BAFzA bis Ende 2012 für die pädagogische Begleitung und die Seminare gewährte, konnte so teilweise für andere Zwecke eingesetzt werden. In Reaktion auf den geringeren Bildungsumfang bei den älteren gegenüber den jüngeren Freiwilligen hat das BAFzA den Zuschuss für die Altersgruppe 27+ zum 1. Januar 2013 um 25 Euro gekürzt. Damit stellt es vermutlich genau die Einsatzstellen vor finanzielle Engpässe, die bereits Bildungskonzepte für die neue Zielgruppe erarbeitet hatten, um den Dienst für alle Generationen als Lerndienst zu gestalten. Gleichzeitig geht hiervon möglicherweise das missverständliche 
Signal aus, die Bildung für die älteren Freiwilligen sei nicht so wichtig. Dabei bestätigen die Experteninterviews, dass sich gerade hier die Chance auftun könnte, bildungsferne Schichten mit passgenauen Angeboten zu erreichen.

Die Entwicklung des BFD zeigt, dass sich auch Personen jenseits der klassischen Mittelschicht grundsätzlich für ein Engagement begeistern lassen. Allerdings engagiert sich diese Klientel vornehmlich „,in eigener Sache“ (Blaschke 2003: 45), das heißt entweder im unmittelbaren Umfeld oder in Verbindung mit einem finanziellen Anreiz. Blaschke spricht von einem „kompensatorische[n] öffentliche[n] Engagement“ (2003: 47), das unmittelbar auf die Verbesserung der eigenen Lebenslage abzielt. Dadurch ergibt sich mitunter eine große Abhängigkeit der Aktiven von ihrem Engagement, da es beispielsweise die einzige Möglichkeit des Zuverdienstes oder der sozialen Teilhabe darstellt. Folglich sind genau diese Personen besonders im Auge zu behalten, damit ihre Abhängigkeit von Einsatzstellen nicht ausgenutzt wird. Es ist deshalb immens wichtig, jeden einzelnen Freiwilligen mit den Bildungsangeboten anzusprechen und eine Partizipation aktiv zu unterstützen.

\section{Experiment geglückt?}

Das Experiment „Altersöffnung“ ist nach unserer Einschätzung noch nicht abgeschlossen. Dass Selbiges nun im Fokus steht, liegt zunächst daran, dass sich ältere Freiwillige in unerwartet großem Umfang für den BFD interessieren.

Die große Resonanz der neuen Zielgruppe hat aber offenbar viele Akteure im BFD recht unvorbereitet getroffen. Die Rahmenbedingungen, besonderen Bedarfe und individuellen Erwartungen dieser Menschen unterscheiden sich maßgeblich von denen der bisherigen Zielgruppen in den JFD und im Zivildienst. Daher muss an einigen Stellen noch nachjustiert werden, damit dauerhaft alle Altersgruppen „durch soziales Engagement positive Erfahrungen sammeln können“ (Deutscher Bundestag 2011: 1). Insbesondere folgende Punkte erscheinen uns besonders dringlich: Zum einen muss ein vergleichbares Bildungsangebot für alle 27+ Teilnehmer im BFD gewährleistet werden; es ist eine Chance, aber auch ein klarer Auftrag ,auch bildungsferne Schichten für Fortbildungsmaßnahmen zu begeistern“ (BMFSFJ 2012a: 15). 
Des Weiteren befindet sich die Klientel $27+$ häufig nicht in einer Übergangsphase und sucht nach einer längerfristigen Einbettung in organisationale Strukturen auch nach dem zeitlich befristeten Freiwilligendienst; darauf sind die meisten Organisationen bisher noch nicht eingestellt. Und schließlich darf ein Freiwilligendienst nicht als arbeitsmarktpolitische Maßnahme wahrgenommen werden. Gerade weil die zeitliche Befristung, der Stundenumfang und die Möglichkeit des Zuverdienstes zu ALG II für die Beteiligten Parallelen aufweisen könnte, muss hier durch eine gelebte Anerkennungskultur, einen verantwortlichen Umgang mit den Freiwilligen und klaren Aufgabenbeschreibungen entgegen gewirkt werden.

\section{Literaturverzeichnis}

Anheier, Helmut K., Annelie Beller, Rabea Haß, Georg Mildenberger und Volker Then (2012): Ein Jahr Bundesfreiwilligendienst. Erste Erkenntnisse einer begleitenden Untersuchung, CSI $\&$ Hertie School of Governance, Berlin.

Backhaus-Maul, Holger, Stefan Nährlich und Rudolf Speth (2011): Der diskrete Charme des Bundesfreiwilligendienstes, in: Aus Politik und Zeitgeschichte, 61. Jg. Heft 48/2011, S. 4653.

Blaschke, Ronald (2003): Arm, arbeitslos und aktiv. Bürgerschaftliches und politisches Engagement armer und arbeitsloser Bürger in eigener Sache, in: Sozial Benachteiligte engagieren sich doch. Über lokales Engagement und soziale Ausgrenzung und die Schwierigkeiten der Gemeinwesenarbeit hrsg. von Chantal Munsch, Weinheim-München, S. 45-78.

Bundesbeauftragter für den Zivildienst (2010): Bericht des Bundesbeauftragten für den Zivildienst zum Prüfauftrag aus der Kabinettsklausur vom 7. Juni 2010, o.O.

Bundesministerium für Familie, Senioren Frauen und Jugend (BMFSFJ) (Hg.) (2011): Zivildienst als Sozialisationsinstanz für junge Männer, Abschlussbericht des Forschungsprojektes, Berlin.

Bundesministerium für Familie, Senioren Frauen und Jugend (BMFSFJ) (2012a): Die neue Kultur der Freiwilligkeit - Perspektiven der Freiwilligendienste in Deutschland. Fachtagung am 19. November 2012 in der Jerusalemkirche, Berlin, Dokumentation, Berlin.

Bundesministerium für Familie, Senioren Frauen und Jugend (BMFSFJ) (2012b): Ziele des Bundesfreiwilligendienstes. Abrufbar unter: http://www.bundesfreiwilligendienst.de/derbundesfreiwilligendienst/ueber-den-bfd.html (Zugriff am 31. Januar 2013).

Deutscher Bundestag (2011): Gesetzentwurf der Bundesregierung. Entwurf eines Gesetzes zur Einführung eines Bundesfreiwilligendienstes. Drucksache 17/4803 vom 17.02.2011.

Engels, Dietrich, Martina Leucht und Gerhard Machalowski (2005): Ergebnisse der Evaluation des FSJ und FÖJ. Abschlussbericht des Instituts für Sozialforschung und Gesellschaftspolitik e.V, Köln-Berlin. 
Voluntaris, Jg. 1, 1/2013, Aufsätze

Gölz, Gisela (2012): Ältere Freiwillige im Bundesfreiwilligendienst. Eine Befragung zu Motivation und Zufriedenheit des ersten Jahrgangs, in: Blätter der Wohlfahrtspflege. Deutsche Zeitschrift für Soziale Arbeit, Jg. 159, 6/2012, S. 213-216.

Institut für Sozialarbeit und Sozialpädagogik e.V. (ISS) (Hg.) (2011): Newsletter der Beobachtungsstelle für gesellschaftspolitische Entwicklungen in Europa. Ausgabe 1/2011: Freiwilligendienste: Nicht nur in Deutschland aktuell, Berlin.

Jakob, Gisela (2011): Freiwilligendienste, in: Handbuch bürgerschaftliches Engagement, hrsg von Thomas Olk und Birger Hartnuß, Weinheim, 185-201.

Jirku, Bernhard (2011): Ist sozial, was Arbeit schafft? Zivilgesellschaft und Soziale Arbeit, in: Forschungsjournal Soziale Bewegungen, 24. Jahrgang, 03/2011, S. 71-76.

Kreuter, Jens (2012): Der Bundesfreiwilligendienst als Lerndienst. BBE-Newletter 11/2012, http://www.b-b-e.de/fileadmin/inhalte/aktuelles/2012/06/nl11_kreuter.pdf (Zugriff am 10. Januar 2013).

Kuhlmann, Klaus (2012): Freiwilligendienste neu organisieren. Der Bundesfreiwilligendienst in der Praxis - „Bufdis“ beim Kreis Lippe, in: Der Landkreis. Zeitschrift für kommunale Selbstverwaltung, 82. Jg., Dezember 2012, S. 696-698.

Schneider, Ulrich (2013): Altersstruktur im Bundesfreiwilligendienst vom 07.01.2013, http://www.ulrich-schneider.de/?p=3293 (Zugriff am 10. Januar 2013).

Schröder, Kristina (2012): Eine neue Kultur der Freiwilligkeit - Perspektiven der Freiwilligendienste in Deutschland, in: Der Landkreis. Zeitschrift für kommunale Selbstverwaltung, 82. Jahrgang, Dezember 2012, S. 686-687.

Strachwitz, Ruprecht Graf (2011): Der neue Bundesfreiwilligendienst. Eine kritische Bewertung aus Sicht der Zivilgesellschaft. Opusculum Nr. 48, Juni 2011, Berlin.

Szymanski, Mike (2011): Engpässe bei Wohlfahrtsverbänden. Der Freiwilligendienst - ein einziger Flop, in: Süddeutsche Zeitung vom 10.07.2011.

Weise, Frank-Jürgen, Hans Heinrich Driftmann, Hans-Ulrich Klose, Jürgen Kluge, Karl-Heinz Lather, und Hedda von Wedel (2010): Bericht der Strukturkommission der Bundeswehr Oktober 2010. Vom Einsatz her Denken. Konzentration, Flexibilität, Effizienz, Berlin. 


\begin{abstract}
Annelie Beller \& Rabea Haß

Experiment volunteer service for all ages: Political goals and non-intended consequences - empirical evidence from the pioneering phase of the federal volunteer service in Germany

Within Europe the German 'Bundesfreiwilligendienst' (BFD, 'Federal Volunteer Service') has been the very first traditional volunteer service programme - given its scope and binding nature - that is open to all generations. The latter was considered a necessity in order to reach a sufficient large number of volunteers that was required for filling the gap of the disestablished civilian service. A continuing demand especially among people aged 27 and over indicates the success of this strategy. In our contribution we focus on this development and investigate the deeper motivation, impetus, and experiences that encourage older people to volunteer. In doing so, we discovered three types of volunteers: Some use the BFD as an opportunity to gain additional job qualifications. Others conceive volunteering as a reasonable alternative to paid labour or active labour-market programmes. Additionally, the programme encourages retired people to engage voluntarily. In conclusion we evaluate in how far political goals have been achieved and which nonintended consequences have been caused.
\end{abstract}

Keywords: Federal Volunteer Service; intergenerational; social engagement policy; qualitative social research; focus group interviews 